\title{
Comparison of Shear Stresses of Different Fluid Flows Arising in the Boundary Layer Theory
}

\author{
Mohammad Riazuddin Molla
}

\author{
Department of Mathematics, Dhaka University, Dhaka-1000, Bangladesh
}

(Received: 28 July 2013; Accepted: 3 February 2014)

\begin{abstract}
The existence of positive solution for the singular second-order nonlinear boundary value problem arising in the boundary layer theory for the strong suction is studied. Then we compared the shear stress of the strong suction with the shear stress of the Homann flow. Also we compared the shear stress of the strong suction with the shear stress of the convergent flow.
\end{abstract}

Keywords : Suction, Positive solution, Homann flow, convergent flow.

\section{Introduction}

The differential equation

$$
f^{\prime \prime \prime}+\alpha f f^{\prime \prime}+\beta\left(1-f^{\prime 2}\right)=0
$$

with boundary conditions

$$
\left.\begin{array}{l}
f(\eta)=f^{\prime}(\eta)=0 \text { at } \eta=0 \\
f^{\prime} \rightarrow 1 \text { as } \eta \rightarrow \infty
\end{array}\right\}
$$

is known as Falkner-Skan boundary layer equation ${ }^{1}$.

For $\alpha=1$ and $\beta=0.5$, the equation (1.1) takes the following form

$$
f^{\prime \prime \prime}+f f^{\prime \prime}+0.5\left(1-f^{\prime 2}\right)=0 \text {, }
$$

with boundary conditions (1.2). This equation represents Homann flow ${ }^{2}$. For $\alpha=0$ and $\beta=1$, the equation (1.1) takes the following form

$$
f^{\prime \prime \prime}-f^{\prime 2}+1=0
$$

with boundary conditions (1.2). This equation represents flow in a convergent channel ${ }^{1}$.

Shin ${ }^{2}$ studied the differential equation (1.3) with boundary conditions (1.2) by using the constructive method such as the method of upper and lower solutions. In this article the constructive method such as the method of upper and lower solutions is also used to study the differential equation

$$
\varphi^{\prime \prime \prime}+\varphi^{\prime \prime}=0
$$

with boundary conditions

$$
\left.\begin{array}{l}
\varphi(\eta)=\varphi^{\prime}(\eta)=0 \text { at } \eta=0 \\
\varphi^{\prime} \rightarrow 1 \text { as } \eta \rightarrow \infty
\end{array}\right\}
$$

which arises in the boundary layer theory for strong suction $^{3}$.

Let the shear stress $g(x)=\varphi^{\prime \prime}(\eta)$ be the dependent variable and the tangential velocity $x=\varphi^{\prime}(\eta)$ be the independent variable. The quantities $x$ and $g$ are called Crocco variables ${ }^{4}$.

Now $\quad g^{\prime}=\frac{d g}{d x}=\frac{\frac{d g}{d \eta}}{\frac{d x}{d \eta}}=\frac{\frac{d}{d \eta}\left(\varphi^{\prime \prime}\right)}{\frac{d}{d \eta}\left(\varphi^{\prime}\right)}=\frac{\varphi^{\prime \prime \prime}}{\varphi^{\prime \prime}}$, which implies

\footnotetext{
*Author for correspondence.e-mail: mriaz_math@yahoo.com
}

$$
g^{\prime} \varphi^{\prime \prime}=\varphi^{\prime \prime \prime}
$$

Differentiating (1.7) with respect to $\eta$ one gets

$$
\varphi^{i v}=g^{2} g^{\prime \prime}+g\left(g^{\prime}\right)^{2} .
$$

As before differentiating (1.5) with respect to $\eta$ one gets

$$
\varphi^{i v}+\varphi^{\prime \prime \prime}=0
$$

This gives

$$
g g^{\prime \prime}+\left(g^{\prime}\right)^{2}+g^{\prime}=0 \text {. }
$$

Now $g(x)=\varphi^{\prime \prime}(\eta)=\frac{d}{d \eta}\left[\varphi^{\prime}(\eta)\right]$.

Therefore

$$
g(1)=\left[\frac{d}{d \eta}\left(\varphi^{\prime}(\eta)\right)\right]_{x=1}=\left[\frac{d}{d \eta}\left(\varphi^{\prime}(\eta)\right)\right]_{\varphi^{\prime}(\eta)=1}=0
$$

Again

$$
g^{\prime}(x)=\frac{d g}{d x}=\frac{\varphi^{\prime \prime \prime}}{\varphi^{\prime \prime}}=\frac{-\varphi^{\prime \prime}}{\varphi^{\prime \prime}}, \text { which implies }
$$$$
g^{\prime}(0)=\left.\frac{d g}{d x}\right|_{x=0}=\left.\frac{d g}{d x}\right|_{\varphi^{\prime}(\eta)=0}=\left.\frac{d g}{d x}\right|_{\eta=0}=\frac{-\varphi^{\prime \prime}(0)}{\varphi^{\prime \prime}(0)}
$$

$$
=-1 \text {. }
$$

Thus by letting $g=\varphi^{\prime \prime}(\eta)$ and $x=\varphi^{\prime}(\eta)$, equation (1.5) with boundary conditions (1.6) can be transformed into a second order singular nonlinear boundary value problem singularity at $x=1$ :

$$
\left.\begin{array}{l}
g g^{\prime \prime}+\left(g^{\prime}\right)^{2}+g^{\prime}=0,0<x<1 \\
g^{\prime}(0)=-1 \text { and } g(1)=0 .
\end{array}\right\}
$$

For $\alpha=1$ and $\beta=0.5$, equation (1.1) with boundary conditions (1.2) can be written as

$$
\left.\begin{array}{l}
g^{2} g^{\prime \prime}-\frac{1}{2}\left(1-x^{2}\right) g^{\prime}=0,0<x<1 \\
g^{\prime}(0)=-0.5 \text { and } g(1)=0 .
\end{array}\right\}
$$

by letting $g=f^{\prime \prime}(\eta)$ and $x=f^{\prime}(\eta)$ and its positive solution has been studied by Shin ${ }^{2}$. Shin ${ }^{2}$ did not state the details of this transformation. In the present paper the details transformation of (1.8) from (1.5) with boundary conditions 
(1.6) are shown using Crocco variables. The nonlinear differential equation (1.1) with boundary conditions (1.2) has been studied by many authors ${ }^{4-14}$ for different values of $\alpha$ and $\beta$ using different methods.

For $\alpha=0$ and $\beta=1$, equation (1.1) with boundary conditions (1.2) can be written as

$$
\left.\begin{array}{l}
g^{2} g^{\prime \prime}+\left(g^{\prime}\right)^{2}-2 x=0,0<x<1 \\
g^{\prime}(0)=-0.86603 \text { and } g(1)=0 .
\end{array}\right\}
$$

by letting $g=f^{\prime \prime}(\eta)$ and $x=f^{\prime}(\eta)$ and its positive solution has been studied by Molla ${ }^{9}$.

The equation (1.8) is equivalent to the nonlinear differential equation (1.5) with boundary conditions (1.6). So the positive solution of $(1.8)$ on $[0,1]$ is equivalent to the the shear stress $f^{\prime \prime}(\eta)$ on $[0, \infty)$.

The main purposes of this article are as follows:

i) To establish the existence of positive solution of (1.8) using the constructive method such as the method of upper and lower solutions.

ii) To compare the shear stress of the strong suction with the shear stress of the Homann flow.

iii) To compare the shear stress of the strong suction with the shear stress of the convergent flow.

Definition 1.1. A function $\alpha_{1} \in C^{2}[0,1]$ is called a positive upper solution of (1.8), if

$$
\begin{aligned}
& \alpha_{1}>0 \text { on }(0,1) \\
& \alpha_{1} \alpha_{1}^{\prime \prime}+\left(\alpha_{1}^{\prime}\right)^{2}+\alpha_{1}^{\prime} \leq 0 \text { on }(0,1) \\
& \alpha_{1}^{\prime}(0) \leq-1 \text { and } \alpha_{1}(1) \geq 0
\end{aligned}
$$

Definition 1.2. A function $\alpha_{2} \in C^{2}[0,1]$ is called a positive lower solution of (1.8), if

$$
\begin{aligned}
& \alpha_{2}>0 \text { on }(0,1) \\
& \alpha_{2} \alpha_{2}^{\prime \prime}+\left(\alpha_{2}^{\prime}\right)^{2}+\alpha_{2}^{\prime} \geq 0 \text { on }(0,1) \\
& \alpha_{2}^{\prime}(0) \geq-1 \text { and } \alpha_{2}(1) \leq 0 .
\end{aligned}
$$

Similar definitions hold for positive upper and lower solutions of a perturbation (2.1) which will be given in the following section.

Definition1.3. A function $g \in C[0,1] \cap C^{2}[0,1)$ is called a positive solution of (1.8), if

$$
\begin{aligned}
& g>0 \text { on }(0,1) \\
& g g^{\prime \prime}+\left(g^{\prime}\right)^{2}+g^{\prime}=0 \text { on }(0,1) \\
& g^{\prime}(0)=-1 \text { and } g(1)=0 .
\end{aligned}
$$

\section{Existence of Positive Solution}

Consider the nonlinear boundary value problem

$$
\left.\begin{array}{c}
g g^{\prime \prime}+\left(g^{\prime}\right)^{2}+g^{\prime}=0,0<x<1 \\
g^{\prime}(0)=-1 \text { and } g(1)=\frac{1}{p}
\end{array}\right\}
$$

for each $p \geq 1$, which may be viewed as a perturbation of (1.8).

To prove the existence of positive solution of (1.8) it is sufficient to established the existence of positive solution of (2.1).

Lemma 2.1. $g_{l p}(x)=0.01 x^{\alpha} \sqrt{1-x}+\frac{1}{p \alpha}$ is a positive lower solution of (2.1), for each $p \geq 1$, where $\alpha=10^{k}$ and $k$ is integer, finite but very large.

Proof. It is clear that $g_{l p}(x)>0$ on $(0,1), g_{l p}^{\prime}(0)=0$, which can be written as $g_{l p}^{\prime}(0)=0 \geq-1, g_{l p}(1)=\frac{1}{p \alpha}$ , which can be written as $g_{l p}(1)=\frac{1}{\alpha p} \leq \frac{1}{p}$ and

$h(x)=g g^{\prime \prime}+\left(g^{\prime}\right)^{2}+g^{\prime}=g_{l p} g_{l p}^{\prime \prime}+\left(g_{l p}^{\prime}\right)^{2}+g_{l p}^{\prime} \geq 0$, for $0<x<1$ and $p \geq 1$.

Thus $g_{l p}$ is a positive lower solution of (2.1). Consequently $g_{l}=0.01 x^{\alpha} \sqrt{1-x}$ is a positive lower solution of (1.8). Notice that if $k=1$ then $h(x)$ is true for $(0,0.9]$, if $k=2$ then $h(x)$ is true for $(0,0.99]$, if $k=3$ then $h(x)$ is true for $(0$ ,0.999] and so on.

Lemma 2.2. $g_{u p}(x)=2 \sqrt{1-x}+\frac{1}{p}$ is a positive upper solution of (2.1) for each $p \geq 1$.

Proof. It is clear that $g_{u p}(x)>0$ on $(0,1), g_{u p}^{\prime}(0)=-1$, which can be written as $g_{u p}^{\prime}(0)=-1 \leq-1$, $g_{u p}(1)=\frac{1}{p} \geq \frac{1}{p}$ and

$g_{u p} g_{u p}^{\prime \prime}+\left(g_{u p}^{\prime}\right)^{2}+g_{u p}^{\prime}$ $=-\left\{2 \sqrt{1-x}+\frac{1}{p}\right\}\left\{0.5(1-x)^{-\frac{3}{2}}\right\}+\frac{1}{1-x}-\frac{1}{\sqrt{1-x}}$ $\leq 0$, for $0<x<1$ and $\mathrm{p} \geq 1$.

Thus $g_{u p}$ is a positive upper solution of (2.1).

Consequently $g_{u}=2 \sqrt{1-x}$ is a positive lower solution of (1.8).

Hence the following Lemma can be formulated from an application of Schauder's Fixed Point Theorem ${ }^{15}$. 
Lemma 2.3. For any $p \geq 1$, there exists a positive solution $g_{p} \in C^{2}[0,1]$ of the problem (2.1) such that $g_{l p} \leq g_{p} \leq g_{u p}$ on $0 \leq x \leq 1$, where $g_{l p}$ and $g_{u p}$ are as given in Lemma 2.1 and Lemma 2.2 respectively.

Lemma 2.4. $g_{p}=(1-x)+\frac{1}{p}$ is a positive solution of (2.1) for each $p \geq 1$.

Proof. It is clear that $g_{p}(x)>0$ on $(0,1)$,

$g_{p}^{\prime}(0)=-1, g_{p}(1)=\frac{1}{p}$ and

$g g^{\prime \prime}+\left(g^{\prime}\right)^{2}+g^{\prime}=g_{p} g_{p}^{\prime \prime}+\left(g_{p}^{\prime}\right)^{2}+g_{p}^{\prime}=0$, for

$0<x<1$ and $p \geq 1$.

Thus $g_{p}$ is a positive solution of (2.1). Consequently $g=1-x$ is a positive solution of (1.8).

Thus by using Lemma 2.3 we can conclude that there exists a positive solution $g \in C^{2}[0,1]$ of the problem (1.8) such that $g_{l} \leq g \leq g_{u}$ on $0 \leq x \leq 1$, where $g_{l}, g$ and $g_{u}$ are as given above.

\section{Results and Discussion}

Here the positive solution of (1.8) is compared with the positive solution of (1.9) and (1.10) studied by Shin $^{2}$ and Molla ${ }^{9}$ respectively. The positive solution $g_{s}$ of (1.9) lies between the positive lower and upper solutions $g_{s l}=0.5(1-x) \quad$ and $\quad g_{s u}=2 \sqrt{1-x}$ respectively obtained by Shin $^{2}$. The positive solution of (1.8) is $g=1-x$. The positive solution $g_{c}=\sqrt{\frac{4-6 x+2 x^{3}}{3}}$ of (1.10) lies between the positive lower and upper solutions $g_{l p}=\sqrt{\begin{array}{l}1.3334-2.0000705 x+0.0000035 x^{2}+ \\ 0.666667 x^{3}\end{array}}$ and $g_{u p}=3 \log (2-x)+3$ respectively obtained by Molla ${ }^{9}$. Molla ${ }^{9}$ also showed that the positive solution converges to the positive lower solution.

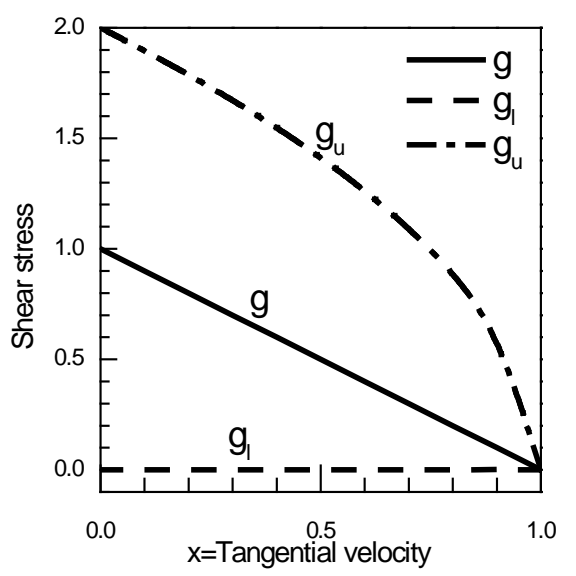

Fig. 1. Different solutions such as (i) positive solution $g$ obtained from present study (ii) positive lower solution $g_{l}$ obtained from present study and (iii) positive upper solution $g_{u}$ obtained from present study.

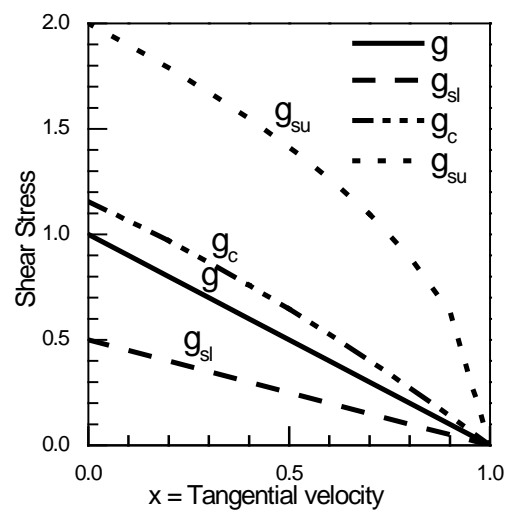

Fig. 2. Different solutions such as (i) positive solution $g$ obtained from present study (ii) positive solution $g_{c}$ obtained by Molla $^{9}$ (iii) positive lower solution $g_{s l}$ obtained by Shin ${ }^{2}$ and (iv) positive upper solution $g_{s u}$ obtained by Shin ${ }^{2}$.

Table 1. Numerical values of different positive, positive lower and positive upper solutions.

\begin{tabular}{|l|l|l|l|l|l|}
\hline$x$ & $g=1-x$ & $g_{c}=\sqrt{\frac{4-6 x+2 x^{3}}{3}}$ & $g_{s l}=\frac{1}{2}(1-x)$ & $\begin{array}{l}g_{l}=0.01 x^{\alpha} \sqrt{1-x} \\
k=1\end{array}$ & $g_{\text {su }}=g_{u}=2 \sqrt{1-x}$ \\
\hline 0 & 1 & 1.1547 & 0.50 & 0 & 2.000 \\
\hline 0.2 & 0.8 & 0.9688 & 0.40 & $9.1589 \mathrm{E}-10$ & 1.789 \\
\hline 0.4 & 0.6 & 0.7589 & 0.30 & $8.1222 \mathrm{E}-10$ & 1.549 \\
\hline 0.6 & 0.4 & 0.5266 & 0.20 & $3.8242 \mathrm{E}-05$ & 1.265 \\
\hline 0.8 & 0.2 & 0.2733 & 0.10 & $4.8019 \mathrm{E}-04$ & 0.894 \\
\hline 1.0 & 0 & 0 & 0 & 0 & 0 \\
\hline
\end{tabular}


From the above figure 1 and Table 1 it is evident that the positive solution $g$ of (1.5) obtained from present study lies on between $g_{l}$ and $g_{u}$. Shin ${ }^{2}$ did not find out the closed form positive solution of (1.9), only mention that there will be exist a positive solution of (1.9) lies on between $g_{s l}$ and $g_{s u}$. Since the positive solution converges to the positive lower solution so the numerical value of the positive solution $g_{s}$ of (1.9) will be very close to the numerical value of $g_{s l}$. Hence from the above figure 2 it is apparent that the positive solution $g$ of (1.5) is less than or equal to the positive solution $g_{c}$ of (1.10) and the positive solution $g_{s}$ of (1.9) less than or equal to the positive solutions $g$ and $g_{c}$ of (1.5) and (1.10) respectively. Notice that equality occurs only when $x=1$. From Table1 it is clear that the shear stress for the strong suction is decreasing uniformly with increasing tangential velocity. On the other hand the shear stress for the convergent flow is decreasing not uniformly with increasing tangential velocity.

\section{Conclusion}

An outcome of the present study is to find a positive lower subtion of equation (1.5). The existence of positive solution of (1.5) is established by using the constructive method such as the method of upper and lower solutions that is also an outcome. There exists a positive solution of (1.5) which lies on between the positive lower and upper solutions is shown. It is found that the shear stress for the strong suction is less than the shear stress for convergent flow with respect to the tangential velocity. It is also found that the shear stress for the Homann flow is less than the shear stress for convergent flow and strong suction with respect to the tangential velocity.

\section{References}

1. Shanti Swarup, 2000. Fluid Dynamics, Krishna Prakashan Media (P) Ltd. Merut, 622, 632,633.

2. Shin J.Y., 1997. A Singular nonlinear differential equation arising in the Homann flow, J. Math. Anal. Appl. 212, 443-451.
3. Schlichting H., and K. Gersten,1999. Boundary Layer Theory, Springer, 113,114.

4. Soewono E., K. Vajravelu and R.N. Mohapatra , 1991. Existence and nonuniqueness of solutions of a singular nonlinear boundary-layer problem, J. Math. Anal. Appl. 159,251-270.

5. Callegari A. and M. Friedman,1968. An analytical solution of a nonlinear, singular boundary value problem in the theory of viscous fluids, J. Math. Anal. Appl. 21, 510-529.

6. Vajravelu K. ,E. Soewono and R.N. Mohapatra,1991.On solutions of some singular, non-linear differential equations arising in boundary layer theory, J. Math. Anal. Appl. 155, 499-512.

7. Molla M.R., 2005. Existence and Uniqueness of positive solution of the suction of the fluid from the boundary layer, $\mathrm{J}$. Math. and Math. Sci, JU, Savar, Bangladesh, 20, 31-40.

8. Molla M.R.,2008. A singular non-linear BVP arising in the boundary layer flow along a flat plate, Ganit: J. Bangladesh Math. Soc. 28, 59-67.

9. Molla M.R., 2012. A singular non-linear boundary value problem arising in a convergent channel, Bangladesh Journal of Physics, 12, 15-26.

10. Molla M.R. and M. Begum, 2012. Existence and Uniqueness of positive solution of the injection of the fluid into the boundary layer.Jahangirnagar J.of Math.and Math.Sciences, 27,103-114.

11. Molla M.R., 2013. An analytic treatment of the Falkner-Skan boundary layer equation. Journal of Science, University of Dhaka, 61(1), 139-144.

12. Molla M.R., M.K. Jaman and M. Hasan, 2011. Comparison of positive solutions for two boundary value problems arising in the boundary layer flow. Journal of Science, University of Dhaka, 59 (2), 167-172.

13. Molla M.R. and S.Banu, 2006. Existence and uniqueness of positive solution of a singular nonlinear BVP. Journal of Science, University of Dhaka, .54 (2), 191-195.

14. Molla M.R. and S. Banu, 2003. Some singular nonlinear BVPS arising in the boundary layer flow. Ganit: Journal of Bangladesh Mathematical Soc., 23, 91-103.

15. Schmidt K.,1970. A nonlinear boundary value problem, J. Differential Equations 7, 527-537. 